\title{
Multi-wavelet level comparison on compressive sensing for MRI image reconstruction
}

\author{
Indrarini Dyah Irawati, Sugondo Hadiyoso, Yuli Sun Hariyani \\ School of Applied Science, Telkom University, Indonesia
}

\begin{tabular}{l} 
Article Info \\
\hline Article history: \\
Received Oct 24, 2019 \\
Revised Feb 17, 2020 \\
Accepted Mar 23, 2020
\end{tabular}

\section{Keywords:}

Compressive sampling

Multi-wavelet

MRI

Reconstruction

\begin{abstract}
In this study, weproposed compressive sampling for MRI reconstruction based on sparse representation using multi-wavelet transformation. Comparing the performance of wavelet decomposition level, which are level 1, level 2, level 3, and level 4 . We used gaussian random process to generate measurement matrix. The algorithm used to reconstruct the image is $\ell_{1}$ norm. The experimental results showed that the use of wavelet multi-level can generate higher compression ratio but requires a longer processing time. MRI reconstruction results based on the parameters of the peak signal to noise ratio (PSNR) and structural similarity index measure (SSIM) show that the higher the level of decomposition in wavelets, the value of both decreases.
\end{abstract}

This is an open access article under the CC BY-SA license.

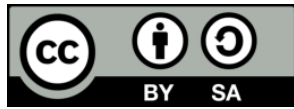

\section{Corresponding Author:}

Indrarini Dyah Irawati,

School of Applied Science,

Telkom University,

Jl. Telekomunikasi, Terusan Buah Batu Bandung 40257, Indonesia.

Email: indrarini@tass.telkomuniversity.ac.id

\section{INTRODUCTION}

Today, there are many electronic devices produce digital information such as image, voice, video and data. Digital imagesincluding digital photography, medical photos (magnetic resonance imaging/MRI, $\mathrm{x}$-ray), seismic image have large size so as require big storage capacity. These images also consume a wide bandwidth when transmitted. MRI medical image has file size about 5-6 MB with 100-1.000 images have contains in series 4-19 [1]. The MRI needs a large enough capacity so that it takes a technique to reduce its capacity thus saving storage space but still ensure the MRI image quality. A new method introduced by Donoho [2] is called compressive sensing (CS) can perform the sampling and reconstruction process simultaneously. CS presents lower signal acquisition rate under the Nyquist rate when the signal known sparse without any loss quality. The signal can be expressed by few samples therefore the signal will be compressed.

There are two properties that must be met at compressive sensing, ie sparsity [3] and incoherency $[4,5]$. Sparsity denotes that a signal can represent in the domain with small number of non-zero samples. Incoherency means that there is no correlation between two different basis, ie the representation base and projection base. An opportunity to achieve sparse signal on the right domain so that CS performs compression with minimal sample with high degree of accuracy.

Compressive sensing application usage has been done in various field such as photography image [6], medical image [7-10], network monitoring [11], holographic microscopy image [12], weather monitoring system [13], and so on. In this paper, we explore the sparsity MRI image representation using wavelet transformation. MRI is a research field that attract the attention of researchers until now. Some research has developed the CS scheme specifically designed for the MR image recovery algorithms [14, 15]. 
Other research, study the sparse MRI representation methods [16-18]. Hot, et al. [16] compared the masked coefficients number of 2D discrete fourier transform (DFT) and 2D discrete cosine transform (DCT) as significant values of the MR image. The experiment result shows that PSNR depends on the mask which groups a number of high frequency coefficients. Guerquin-Kern, et al. [17] combined sampling method with arbitrary $\mathrm{k}$-space trajectories using wavelet and iterative shrinkage/thresholding algorithm (ISTA) to accelerate MRI reconstruction. The proposed method is superior to total varian (TV) regularization in terms of image quality. In [18], authors proposed four level wavelet-encoded CS (WE-CS) to recover MR images using reduced samples. This method improves the performance and reduces the sampling time compared to fourier-encoded CS (FE-CS). MRI research on wavelet-based image reconstruction has actually been carried out by several researchers. Some researchers have proposed methods or algorithms for MRI image reconstruction specifically wavelet-based. Research by Chen and Huang [19], proposed a wavelet tree structure to improve the performance of conventional compressed sensing MRI. Research by Ragab et. al [20], proposed compressive sensing MRI reconstruction using empirical wavelet transform. This research claims that the proposed method outperforms the state-of-the-art methods in terms of signal-to-noise ratio and structure similarity metrics. Similar research by Ragab et. al [21], simulates the dual tree complex wavelet transform method on CS-MRI. This research has a better SNR performance compared to MRI wavelet tree sparsity. Lai et. al [22] has proposed compressed sensing MRI using graph-based redundant wavelet transforms. However, this proposed method is only able to produce an SNR of less than $20 \mathrm{~dB}$ at a $20 \%$ sampling rate. Zhu et. al [23] has proposed CS MRI reconstruction using a DWT dual-density dual-tree complex. This method modifies conventional wavelet decomposition to get more detailed sub-bands that are expected to reduce aliasing artifacts. These reviews provide a thought that CS techniques will become an interesting topic in the future to overcome the limitations of memory storage. The main issue and also the problem of implementing CS especially on images is how to get a high compression ratio and PSNR with simple techniques and produce high accuracy. This paper proposes a compressive sensing with the multi-wavelet technique which is applied to MRI images. This method is combined with the $\ell_{1}$ norm to reconstruct the image algorithm. This proposed method becomes the state of the art of the proposed method in related research above. The aim is to analyze the effect of multi wavelets on the performance of the proposed method so that the best approach can be applied in the future. The structure of the paper is arranged as follows, section 2 presents the proposed method for MRI reconstruction system. The experimental results and analysis are provided in section 3 . In section 4 is shown summary and conclusions, also further research.

\section{RESEARCH METHOD}

The proposed of compressive sensing simulation modelingfor the MRI reconstruction is shown in Figure 1. MRI input is an image with format extensions such as .png and .bmp. Sparsity base aims to produce sparse image which has non-zero values mouth slightly. In this research, we explore the sparsity by comparing the level of wavelet transformation. We also examine the effect of level wavelet transformation such as level 1, level 2, level 3 and level 4. We take a $M \times N$ mesurement matrix $(\Phi)$ that produced from random gaussian process. Reconstruction algorithms serves to restore the compressed image. We use $\ell_{1}$ norm for reconstructing [24]. MRI output is the result of the inverse wavelet transform.

In DWT 2D image used by the filter process using wavelet bases in rows and columns that make the matrix approximation and detail. The decomposition process is tiered so-called multi wavelet as shown in Figure 2. In the matrix approximation done back in the filter process to produce a matrix approximation and detail at the next level. In this study a comparison effect level wavelet used against the parameters measured. Wavelet is a more efficient method for estimating original images with a small number of basic elements. In mathematical equations, wavelet transform on the function $\mathrm{x}(\mathrm{t})$ is interpretation of these functions into a set of basis functions below.

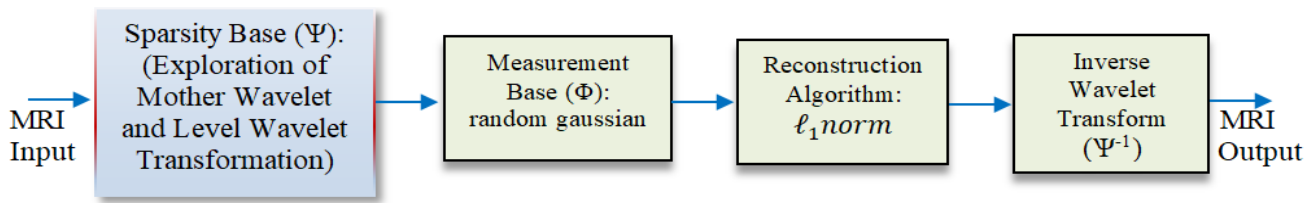

Figure 1. The proposed of compressive sensing simulation modelingfor the MRI reconstruction

$$
\mathrm{X}_{\omega}(t)=\int_{-\infty}^{\infty} x(t) \cdot \Psi_{a, b}(t) d t
$$


where $\Psi_{a, b}(t)$ is a basic function, which is a bandpass version of an enlarged and shifted time signal $\Psi(\mathrm{t})$. The signal $\Psi(\mathrm{t})$ is named the mother wavelet and expressed as follows:

$$
\Psi_{a, b}=\frac{1}{\sqrt{a}} \Psi\left(\frac{t-b}{a}\right)
$$

where $\mathrm{a}$ is the enlargement parameter and $\mathrm{b}$ is the shift parameter.

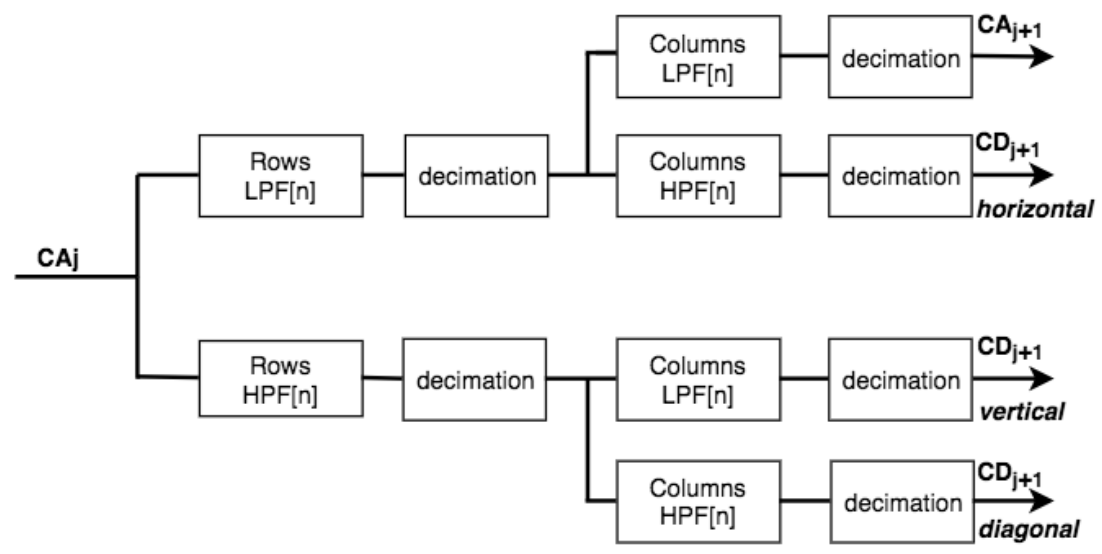

Figure 2. 2D wavelet decomposition [25]

Donoho introduces compressive sensing as a new method of signal processing [2]. The main idea is to take the signal with less sample below Nyquist theory where the sampling results meet the original sparse signals. The original has a sparse representation thereof if it is expressed in the appropriate base. The mathematical equations for a vector $x \in R^{n}$ is represented using the wavelet basis $\Psi=\left[\Psi_{1} \Psi_{2} \ldots \Psi_{N}\right]$, vector $\mathrm{x}$ can be formulated [11].

$$
x=\sum_{i=1}^{N} s_{i \Psi_{i}}(t)
$$

wheres is the coefficient of $\mathrm{x}$ obtained from $s_{i}=\left\langle x, \Psi_{1}\right\rangle$.

In matrix form can be written:

$$
x=\Psi S
$$

where $\Psi$ an $\mathrm{N} x \mathrm{~N}$ matrix, $\mathrm{x}$ and $\mathrm{s}$ is a column vector $\mathrm{N} \times 1$.

Signals $\mathrm{x}$ expressed $\mathrm{K}$-sparse if only $\mathrm{K}$ from coefficients of $s$, when the value is not zero while the $(\mathrm{N}-\mathrm{K})$ coefficient is zero. In compressive sensing is required $M$ sample of the signal, where $K<M \ll N$. As shown in Figure 1, $\mathrm{y}$ is a measurement vector with length $(\mathrm{M})$, then can be expressed in the equation:

$$
y=\Phi x=\Phi \psi s
$$

with $\mathrm{y}$ is the output signal that has been compressed, $\Phi$ is the projection matrix with size $\mathrm{Mx} \mathrm{N}, \Psi$, a base matrix with size $\mathrm{N} \times \mathrm{N}$, and $\theta=\Phi \psi$ is the matrix $\mathrm{M} \times \mathrm{N}$.

Based on the equation 5, to get the compressed signal $\mathrm{y}$, the required measurement matrixM $\mathrm{x} \mathrm{N}$. The measurement matrix used is a gaussian random matrix. The smaller the value of $\mathrm{M}$ (measurement rate) then the resulting matrix size is also smaller. In this study measured the effect of the value of $\mathrm{M}$ or the number of sampling of the measured parameters namely compression ratio (CR).

After getting the uncompressed signal (y), then do the reconstruction process to obtain the original image. Some reconstruction algorithm that can be used include norm-order one, orthogonal matching pursuit based greedy algorithm, and others.In this experiment, the algorithm first order norm for reconstruction poses. Image reconstruction results will be compared with the original image and then calculated PSNR and SSIM. Another measurement parameter is the duration of the process named processing time. 


\section{RESULTS AND ANALYSIS}

In this study, we measured several parameters, such as PSNR, SSIM, CR, and processingtime for the use of wavelet and multi wavelet. Matrix measurement used is a gaussian random matrix with testing for $\mathrm{M}$ different values of $10 \%$ to $90 \%$. While the reconstruction algorithm used is $\ell_{1}$ norm. Tests performed on the images consist of cephal MRI with a size of 256 × 256 and brain MRI transversal with size $350 \times 227$. Because the matrix is used random matrix, then tested 5 times and then calculated the average for each parameter.

The PSNR parameter test results can be seen in Figure 3. The test is performed for cephal MRI. PSNR illustrated the quality of the reconstructed image. The test results show that the greater the measurement rate,the PSNR value increases. Experiments at the DWT decomposition level show that the more decomposition levels, the PSNR is decreasing. According to [26], the image quality is declared good when it has a PSNR $\geq 30 \mathrm{~dB}$. According to Figure 3, DWT level 1 and level 2 have good image quality at measurement rate $\geq 20 \%$, whereas DWT level 3 and level 4 have good quality at measurement rate $\geq 80 \%$.

SSIM is a parameter that shows the level of resemblance between the original image and the reconstructed image. Figure 4 presents the SSIM values at different DWT decomposition levels on cephal MRI, which illustrates the greater the value of the measurement rate the higher the SSIM value. From the test results, level 1 decomposition has the highest SSIM compared to level 2, level 3, and level 4, almost all of the measurement rate.

$\mathrm{CR}$ is used to show the performance of the compression algorithm in the relative reduction in the size of the resulting data compressionwhich expressed as uncompressed size division by compressed size. Figure 5 shows CR on cephal MRI, which illustrates that the greater the measurement rate, the smaller the CR. When compared between levels of DWT decomposition, the greater the level of decomposition, the CR is increasing. In this case, CS on DWT with a high level of decomposition is suitable for obtaining large CRs thus saving storage space.

Figure 6 shows the processing time for cephal MRI. The processing time is proportional to the complexity of the algorithm is executed. Multi tiered process on wavelet scheme requires a longer time than the wavelet level 1 . The processing time at DWT level 4 is the longest compared to the others.

Based on PSNR, SSIM, CR, and processing time metrics it can be explained that PSNR is proportional to SSIM and inversely proportional to CR and processing time. The higher level of DWT decomposition will result in high CR with the risk of a long processing time, but reduces the accuracy seen from the PSNR and SSIM parameters. In these experiments, the DWT decomposition level 2 is suitable for obtaining high CR while still obtaining good reconstruction image quality with a minimum measurement rate of $20 \%$.

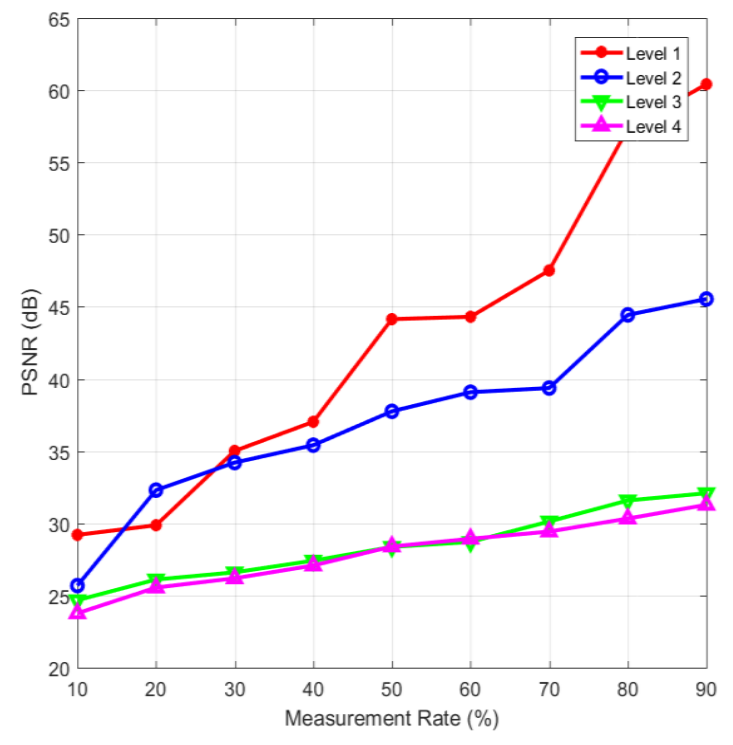

Figure 3. PSNR for measurement rate $[10 \%-90 \%]$ at different DWT decomposition levels

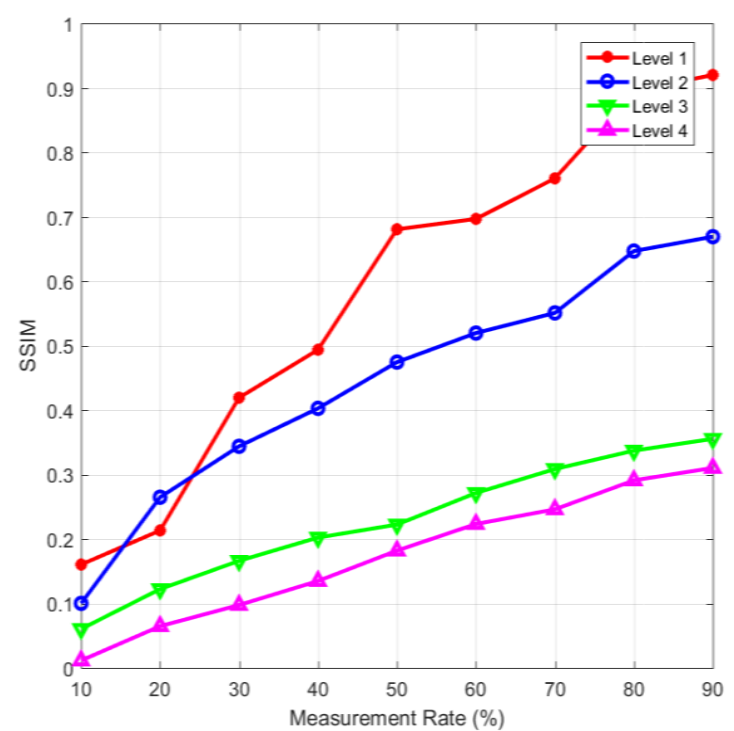

Figure 4. SSIM for measurement rate [10\%-90\%] at different DWT decomposition levels 


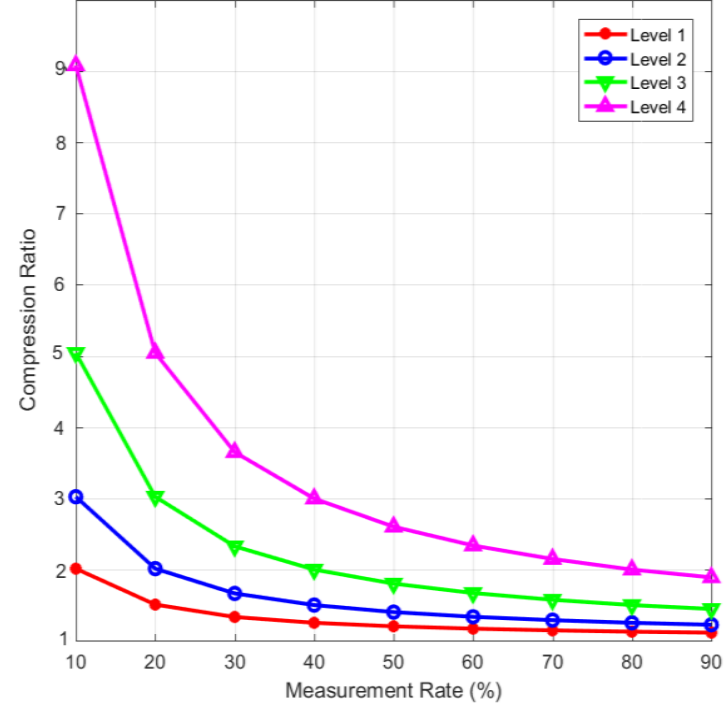

Figure 5. CR for measurement rate [10\%-90\%] at different DWT decomposition levels

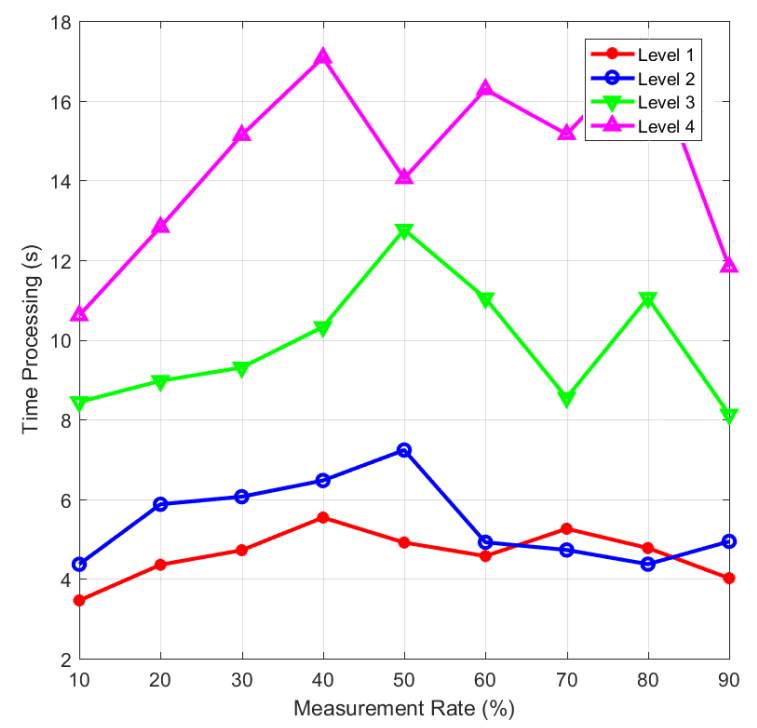

Figure 6. Time of processing for measurement rate [10\%-90\%] at different DWT decomposition levels

Figure 7 and Figure 8 show the comparison between the original image with the reconstruction image at DWTdecomposition level 1 (b) and (c) and DWT decomposition level 2 (d) and (e) for measurement rates of $50 \%$ and $90 \%$. Figure 7 for cephal MRI while Figure 8 for brain MRI transversal. According to Table 1, for measurement rate 50\% in cephal MRI with DWT decomposition level 2 is obtained PSNR=38.12dB, $\mathrm{SSIM}=0.486$, and $\mathrm{CR}=1.85$. When seen in plain view, the results of reconstruction at measurement rate $=50 \%$ are still unclear, different from the results of DWT decomposition level 1 for the same measurement rate. Image obscurity was also seen in experiments on brain MRI transversal, with the value of each parameter as follows: $\mathrm{PSNR}=39.89 \mathrm{~dB}, \mathrm{SSIM}=0.499$ and $\mathrm{CR}=2.11$.

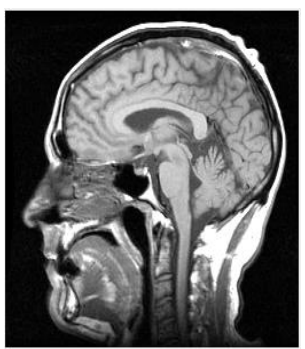

(a)

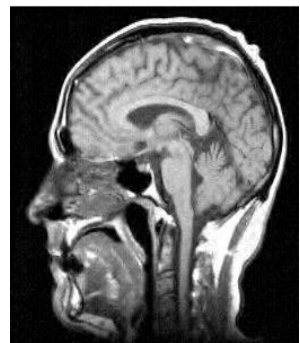

(b)

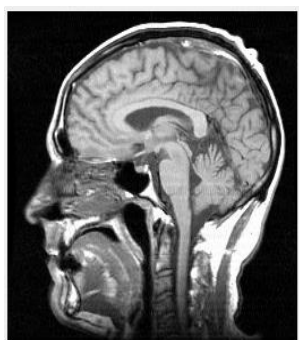

(c)

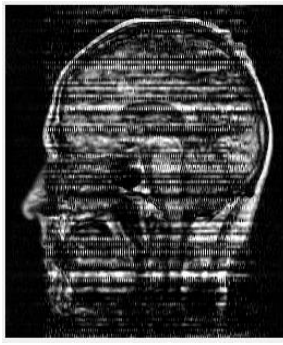

(d)

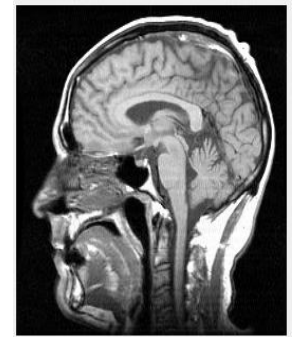

(e)

Figure 7. Cephal MRI 256x256, (a) Original image, (b) Reconstruction image using level 1 (measurement rate $=50 \%$ ), (c) Reconstruction image using level 2 (measurement rate $=90 \%$ ), (d) Reconstruction image using level 2 (measurement rate $=50 \%$ ), (e) Reconstruction image using level 2 (measurement rate=90\%) 


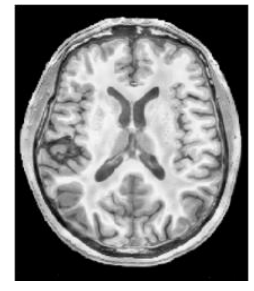

(a)

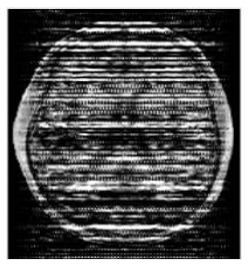

(d)

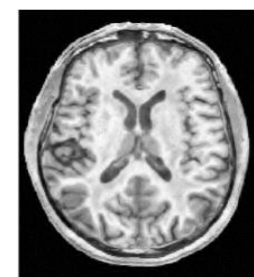

(b)

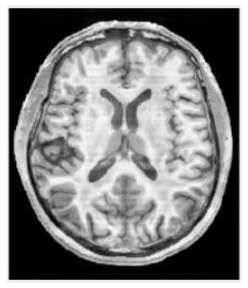

(c)

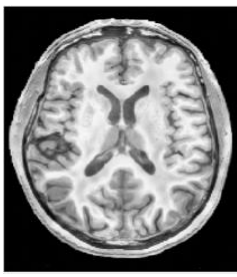

(e)

Figure 8. Brain MRI transversal 350x227, (a) Original image, (b) Reconstruction image using level 1 (measurement rate $=50 \%$ ), (c) Reconstruction image using level 1 (measurement rate $=90 \%$ ),

(d) Reconstruction image using level 2 (measurement rate=50\%), (e) Reconstruction image using level 2 (measurement rate $=90 \%$ )

Table 1. PSNR, SSIM, CR for different level decomposition for measurement rate $=50 \%$

\begin{tabular}{ccccccc}
\hline \multirow{2}{*}{ Parameter r } & \multicolumn{3}{c}{ Cephal MRI } & \multicolumn{3}{c}{ Brain MRI } \\
\cline { 2 - 7 } & PSNR (dB) & SSIM & CR & PSNR (dB) & SSIM & CR \\
\hline Level 1 & 44.59 & 0.689 & 2.76 & 47.76 & 0.712 & 3.01 \\
Level 2 & 38.12 & 0.486 & 1.85 & 39.89 & 0.499 & 2.11 \\
Level 3 & 28.19 & 0.243 & 1.46 & 32.97 & 0.313 & 1.54 \\
Level 4 & 28.15 & 0.198 & 1.23 & 32.67 & 0.245 & 1.33 \\
\hline
\end{tabular}

\section{CONCLUSION}

In this paper, several conclusions are, the higher the number of measurement rate the greater PSNR and SSIM value are generated, the smaller the CR is obtained, and the longer the processing time is needed. The use of multi wavelet scheme is able to increase the CR but reduce PSNR and SSIM and delayed processing time. In this experiment, DWT level 2 is recommended for compression with a measurement rate $>50 \%$ to get a good MRI reconstruction quality.

Future research is to develop a method for obtaining sparsity based on wavelet technique by forming a new matrix containing wavelet coefficients with significant values. In addition, we will explore the effects of using other measurement matrices such as Bernoulli, Binomial, the use of proposed methods for color MRI or dynamic MRI, observing the effect of using other reconstruction algorithms.

\section{REFERENCES}

[1] "Magnetic Resonance Imaging (MRI)," 2017, [Online], Available: http://www.telemedproviders.com/telemedicinearticles/91-magnetic-resonance-imaging-mri.html

[2] D. L. Donoho, "Compressive Sampling," IEEE Transaction on Information Theory, pp. 1289-1306, 2006.

[3] E. J. Candes and M. B. Wakin, "An Introduction to Compressive Sampling," in IEEE Signal Processing Magazine, vol. 25 , no. 2, pp. 21-30, 2008.

[4] E. Candes and M. Walkin, "An introduction of compressive sampling," Signal Processing Magazine, no. 2, vol.25, pp. 21-30, 2008

[5] E. J. Candes, J. Romberg and T. Tao, "Robust uncertainty principles:Exact signal reconstruction from highly incomplete frequency information," In IEEE Transaction Information Theory, vol. 52, no. 2, pp. 489-506, 2006.

[6] I. D. Irawati and A. B. Suksmono, "Image Reconstruction Based on Compressive Sampling using IRLS and OMP Algorithm," JurnalTeknologi, vol. 78, no. 5, pp. 309-314, 2016.

[7] M. Lustig, D. Donoho, J. Santos and J. Pauly, "Compressed sensing MRI," in IEEE Signal Processing Magazine, vol.25, no. 2, pp. 72-82, 2008.

[8] M. Lakshminarayana and M. Sarvagya, "OFCS: Optimized framework of compressive sensing for medical images in bottleneck network condition," International Journal Electrical, Computer Engineering, vol. 8, no. 5, pp. 2829-2838, 2018. 
[9] M. Lakshminarayana and M. Sarvagya, "MICCS: A novel framework for medical image compression using compressive sensing," International Journal Electrical, Computer Engineering, vol. 8, no. 5, pp. 2818-2828, 2018.

[10] W. Swastika and H. Haneishi, "Compressed sensing for thoracic MRI with partial random circulant matrices," TELKOMNIKA Telecommunication Computing Electronics and Control, vol. 10, no. 1, pp. 147-154, 2012.

[11] I. D. Irawati, A. B. Suksmono and I. J. M. Edward, "Missing Internet Traffic Reconstruction using Compressive Sampling," International Journal of Communication Networks and Information Security (IJCNIS), vol. 9, no.1, pp. 1-10, 2017.

[12] A. B. Suksmono and A. Hirose, "Reconstruction of Holographic Microscopy Images Based on Matching Pursuit on A Pair of Domains," Internatiol Journal on Electrical Engineering and Informatic, vol. 8, no. 3, pp. 553-566, 2016.

[13] R. Sustika and B. Sugiarto, "Compressive sensing algorithm for data compression on weather monitoring system," TELKOMNIKA Telecommunication Computing Electronics and Contro., vol. 14, no. 3, pp. 974-980, 2016.

[14] S. Ma, W. Yin, Y. Zhang and A. Chakraborty, "An Efficient Algorithm for Compressed MR Imaging using total variation and wavelets," InConference: Computer Vision and Pattern Recognition, 2008.

[15] B. Jelena, "Comparison of algorithms for compressed sensing of magnetic resonance images," In 4th Mediterranean Conference on Embedded Computing (MECO), pp. 303-306, 2015.

[16] E. Hot and P. Sekulić, "Compressed sensing MRI using masked DCT and DFT measurements," In 4th Mediterranean Conference on Embedded Computing (MECO), pp. 323-326, 2015.

[17] M. Guerquin-Kern, M. Haberlin, K. P. Pruessmann and M. Unse, "A Fast Wavelet-Based Reconstruction Method for Magnetic Resonance Imaging," IEEE Transaction Medical Imaging, vol. 30, no. 9, pp. 1649-1660, 2011.

[18] Z. Liu, B. Nutter and S. Mitra, "Compressive sampling in fast wavelet-encoded MRI," In IEEE Southwest Symposium on Image Analysis and Interpretation, pp. 137-140, 2012.

[19] C. Chen and J. Huang, "Exploiting the wavelet structure in compressed sensing MRI," Magn. Reson. Imaging, vol. 32, no. 10, pp. 1377-1389, 2014.

[20] M. Ragab, O. A. Omer, and M. Abdel-nasser, "Compressive sensing MRI reconstruction using empirical wavelet transform and grey wolf optimizer," Neural Comput. Appl., vol. 7, pp. 1-20, 2018.

[21] M. Ragab, O. A. Omer and H. S. Hussien, "Compressive sensing MRI using dual tree complex wavelet transform with wavelet tree sparsity," In34th National Radio Science Conference (NRSC), pp. 481-486, 2017.

[22] Z. Lai, X. Qu, et. al., , "Image Reconstruction of Compressed Sensing MRI Using Graph-based Redundant Wavelet Transform," Med. Image Anal., vol. 27, pp. 93-104, 2015.

[23] Z. Zhu, K. Wahid, P. Babyn, and R. Yang, "Compressed Sensing-Based MRI Reconstruction Using Complex Double-Density Dual-Tree DWT,” International Journal ofBiomedical Imaging, vol. 5, pp. 1-12, 2013.

[24] E. Candes and J. Romberg, "11-magic: Recovery of Sparse Signals via Convex Programming," [Online] available: http://www. acm. caltech. edu/11magic/downloads/11magic. pdf 4, 14., accessed at: october 13, 2015, 2005.

[25] A. D. Sappa, J. A. Carvajal, C. A. Aguilera, M. Oliveira, D. Romero, and B. X. Vintimilla, "Wavelet-based visible and infrared image fusion: A comparative study," Sensors,vol. 16, no. 6, pp. 1-15, 2016.

[26] Y. C. Lin, D. Varodayan and B. Girod, "Image Authentication Based on Distributed Source Coding," In IEEE International Conference on Image Processing, 2007.

\section{BIOGRAPHIES OF AUTHORS}
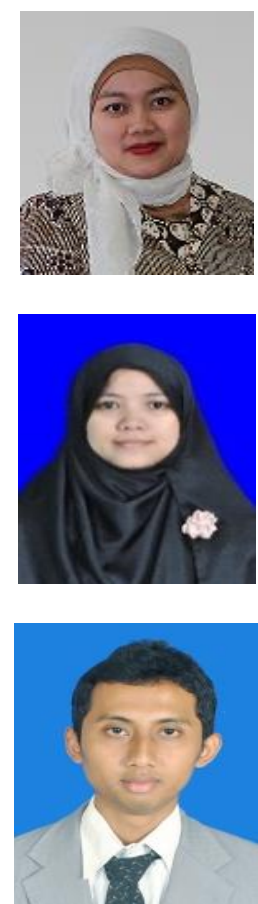

Indrarini Dyah Irawati, obtained a bachelor and master degree in Electrical Engineering at Telkom University, Bandung, Indonesia and doctoral degreein the School of Electrical and Information Engineering, Institute of Technology Bandung. She joined as a Lecturer at Telkom Applied Science School, Telkom University, since 2007. She is currently a member of the Association for Computing Machinery (ACM) and the International Association of Engineers (IAENG). Her main research interests are in the areas of compressive sensing, watermarking, signal processing, and computer networking.

Yuli Sun Hariyani received the Master.in Electrical-Telecommunication Engineering from Telkom University, Bandung, Indonesia. She joined as a Lecturer of Telkom University, in 2010. Her research interests are compressive sampling, Image Processing and Biomedical Engineering

Sugondo Hadiyoso received the Master.in Electrical-Telecommunication Engineering from Telkom University, Bandung, Indonesia in March 2012. He joined as a Lecturer in the department of Electronics and Communication Engineering of Telkom University, in 2010. where he is currently members of Biomedical Instrumentation Research Group in Telkom Univesity. His research interests are wireless sensor network, embedded system, logic design on FPGA and biomedical engineering. 